\title{
AN EXPERIMENTAL CENTER FOR Mesoscale OCEAN PREDiction
}

By John A. Leese, M. Stephen Foster and James $H$. Corbin

\section{. . . a sophisticated}

data-base

management system is a high priority. . . in a numerical ocean modeling and prediction system.
$\mathrm{T}$ HE NEEDS of the naval and academic community to expand their modeling activities were identified in an Ocean Prediction Workshop during 1986 (Mooers et al., 1986): 1) an openly shared hierarchy of well-documented models; 2) direct access to computational resources; 3) improvement of scientist-to-scientist communications through standardized procedures for model testing and validation; and 4) various kinds of certified data sets. The Institute for Naval Oceanography (INO) response to these needs was to implement an Experimental Center for Mesoscale Ocean Prediction (ECMOP). This is a specialized computational center to facilitate the development, demonstration, and evaluation of a mesoscale ocean-prediction capability.

An early planning study (INO, 1988) investigated the functional components of an experimental center. This study concluded that the best overall strategy for ECMOP was to take advantage of the infrastructure provided by the Naval Oceanography Command for real-time operations and to provide capability for validation and evaluation of ocean models. Another feature was a conceptual design for ECMOP. The major functions within the framework of a numerical ocean prediction system should be structured with sufficient modularity to provide flexibility for modification as needed to conduct evaluation of ocean modeling and prediction. Figure 1 depicts the major features of such a modular prediction system (INO, 1988). This figure shows the many places where different data sets are needed or accumulated. It also shows clearly why a sophisticated data-base management system is a high priority for early implementation in a numerical ocean modeling and prediction system.

J.A. Leese, M.S. Foster, and J.H. Corbin. Institute for Naval Oceanography, Stennis Space Center, MS 39529, USA. The Institute for Naval Oceanography is operated by the University Corporation for Atmospheric Research (UCAR) for the Naval Research Laboratory (NRL).

\section{Experimental Center for Mesoscale Ocean Prediction as a Specialized Facility}

ECMOP has set three major long-term goals:

1) provide the infrastructure and support needed for research and development in ocean modeling and prediction. The emphasis is on data management, visualization, and evaluation;

2) adopt the technology and methodology in these areas for applications in operational ocean predictions; and

3) continue to update the technology and methodology for the scientific community.

To establish a high-quality, wide-spectrum data base and software library for the oceanic modeling community, ECMOP has adopted two paradigms. ECMOP will use existing data in the National Oceanic and Atmospheric Administration (NOAA) and the Navy archives so that the ECMOP resources can be concentrated on establishing special data sets for research and evaluation of ocean modeling and prediction. ECMOP will create incentives for investigators to submit their data to the data pool. The chief benefit to contributors is the use of the data sets contributed by other investigators.

An ECMOP User Support and Model Library will place heavy dependence on sources outside ECMOP. To take advantage of the many potential sources of software and achieve a cost-effective service, ECMOP will classify several different levels of applications software and models in the library. The user support, including documentation and user guides, will be according to the classification level for each of the software items. A scientific and technical advisory group has been formed to provide advice and assistance.

\section{Evolutionary Development of the Experimental} Center for Mesoscale Ocean Prediction

An early objective for ECMOP was to develop. demonstrate, and evaluate an initial ocean-prediction system based on existing capability. Stability and flexibility were needed to provide a 
learning experience and to demonstrate the validity of a specialized facility for evaluation of ocean model and prediction systems. Our design for a case study took the modular concept, previously shown in Figure 1, and applied state-of-the-art technology in data-base management. Together with existing components for each of the modules, a system was constructed and named the Primitive Equation Data Assimilating Model (PEDAM). The ocean model was specifically configured for the Gulf of Mexico (GOM).

The ECMOP/PEDAM (GOM) ocean prediction system is depicted schematically in Figure 2. A four-dimensional data-assimilation scheme (Derber and Rosati, 1989) was combined with the Princeton ocean general circulation model (Blumberg and Mellor, 1987) and was applied to the Gulf of Mexico by Drs. L. Kantha and R. Passi at the INO. An initial set of statistical and physical measures based on the results of the Model Evaluation Criteria Colloquium (Willems, 1989) was used to form the Verification Module (Anand et al., 1991). The data management portion of the system was comprised of a proprietary data-base management system and the Naval Environmental Operational Nowcast System (NEONS). The National Center for Atmospheric Research (NCAR) Graphics package was employed to fulfill visualization objectives.

Real data in the form of conventional expendable bathythermograph (XBT) observations, along with satellite-derived sea-surface temperature and sea-surface height measurements derived from the Geodetic Earth Orbiting Satellite (GEOSAT) altimeter data for the period November 1986 to April 1987, were used as input data to PEDAM (Ko, unpublished observations). Model output fields were evaluated in both nowcast and hindcast modes (Waters et al., 1990). The case study clearly demonstrated that users of this specialized facility could exploit the system's capabilities after a short orientation period.

\section{A Prototype Modular System}

The lessons learned from the ECMOP case study were incorporated into a PROTotype ECMOP Modular System (PROTEMS). The major focus of the PROTEMS is a graphical-user interface that gives both the user and the designer greater flexibility but still maintains ease of learning and use. The primary function of PROTEMS is to access data sets (historical, climatological, etc.) for visualization or evaluation.

PROTEMS facilitates a single package solution to the problem of mastering the details of numerous software applications during model development and assessment. All the necessary development tools have been made available through a graphical-user interface. All software elements within PROTEMS have one critical factor in common-their ability to interface with the

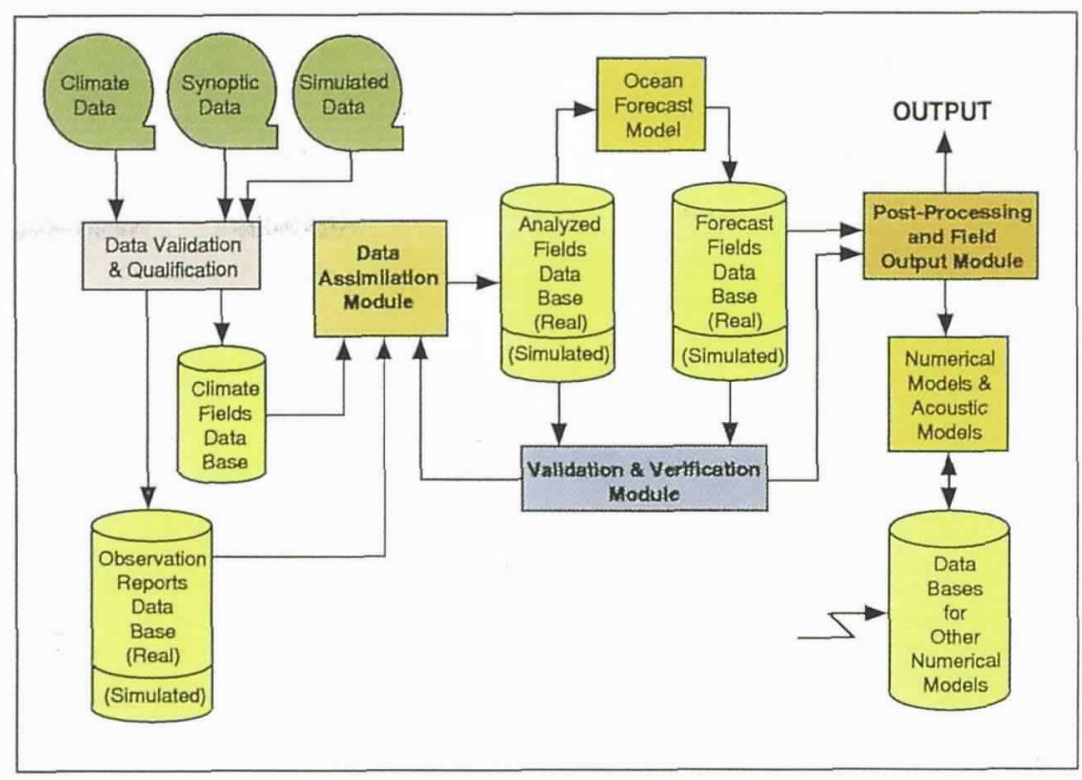

Fig. 1: Components of an ocean prediction system depicting input data and ocean model output fields as separate entities.

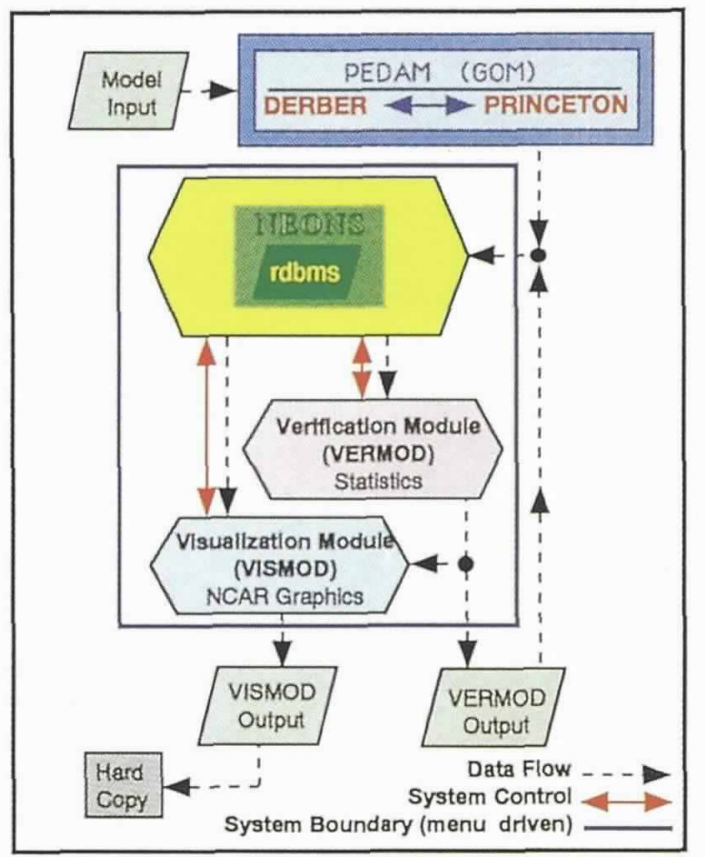

Fig. 2: Modular flow diagram for the ECMOP system as configured during 1990 for the Primitive Equation Data Assimilation Model (PEDAM) case study for the Gulf of Mexico (GOM). The PEDAM case study successfully demonstrated the utility of the ECMOP modular concept in constructing a system for assessing ocean model performance and displaying the output. Control linkage was weak at this early stage in system development.

$\mathrm{C}$ and Fortran programming languages. Currently, there are three principal modules (Fig. 3) within PROTEMS: 1) the Data Management Module
The primary function

. . . is to access data sets . . . for evaluation. visualization or 


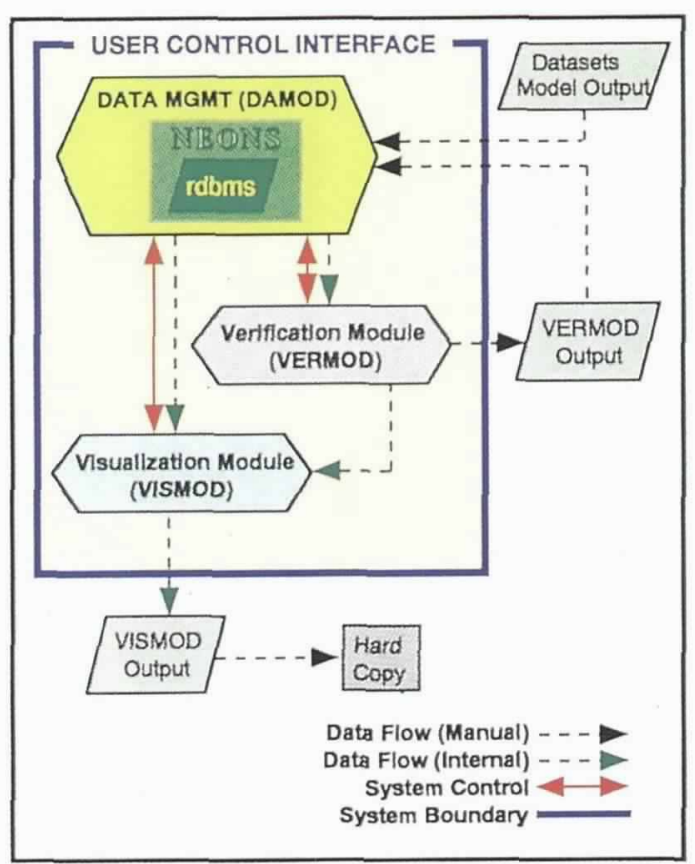

Fig. 3: The Prototype ECMOP Modular System (PROTEMS) as currently implemented. A Graphical User Interface (GUI) has been added, which interprets and acts upon user commands and which controls internal data flow. Future plans include addition of an acoustics module, a data browser and, most importantly, extension of the GUI to incorporate linkage with an ocean model and control of module output.

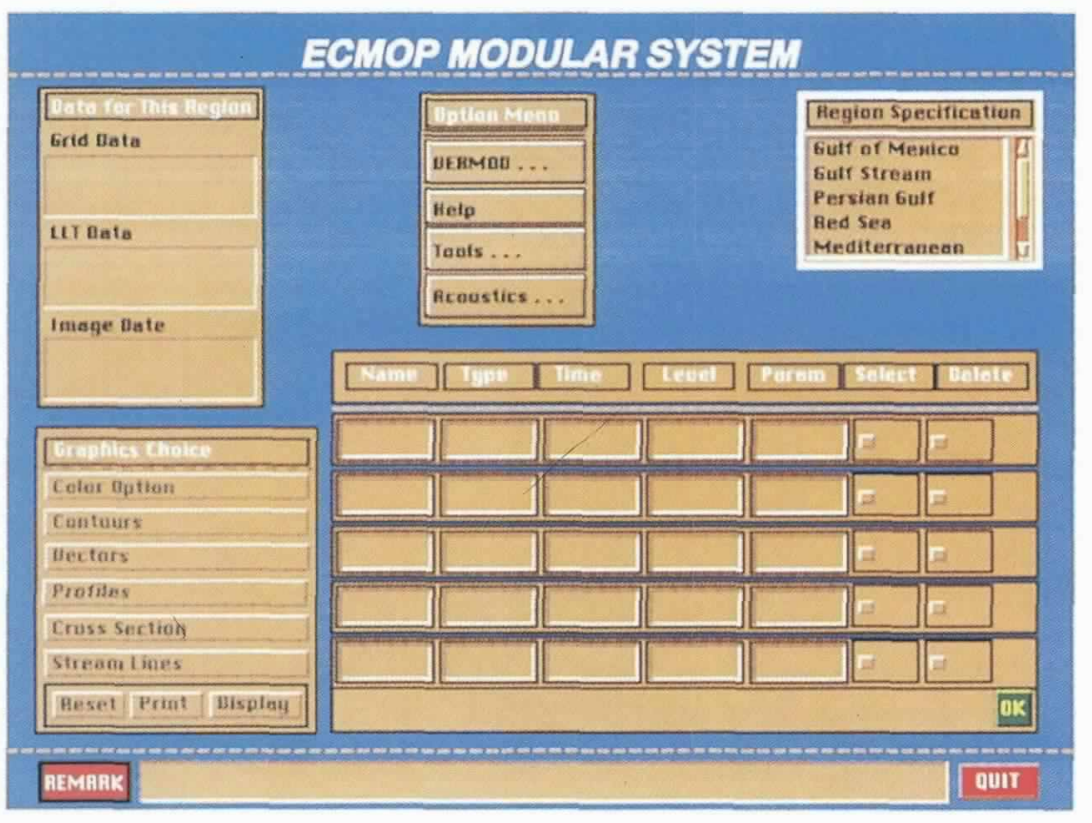

Fig. 4: Initialization screen for PROTEMS (current implementation). User interaction begins in the upper right corner with selection of a region. Region selection initiates a search of the data base for data types appropriate to the selected region. The data types are listed in the box at the upper left for user selection. Those data sets selected by the user are then displayed in the matrix at lower right for further parameterization. When this step is completed, the user may pass the data to VERMOD (verification module; top center) or exercise the graphics choices shown at lower left.
(DAMOD); 2) the Verification Module (VERMOD); and 3) the Visualization Module (VISMOD). These modules, when interfaced with a user-supplied ocean model and a data-assimilation technique, comprise an ocean prediction system. The INO-designed graphical-user interface (Fig. 4) upgrades the original menu-driven version developed for the PEDAM (GOM) case study.

Users indicate their data requirements to the DAMOD by selecting the geographical region, model type, physical parameter(s), levels, and time period from the opening display screen and subsequent "pop-up" displays. Selections are made with a "mouse" input device. These straightforward, highly visual steps require minimal skill even for an inexperienced computer user. As many as five separate data groups may be chosen before invoking evaluation or graphics options.

Assessment of ocean model performance is the principal justification for PROTEMS. VERMOD statistical tools currently require data in grid format. VERMODs root-mean-square-error capability accommodates weighting functions and employs a widely accepted derivation (Wilmot et al., 1985) for its decomposition into systematic (bias) and unsystematic (exactness) error components. Other standard statistical routines include pattern correlation, skewness, and kurtosis.

Visual display of model output and derivative data give the ocean modeler a clearer understanding of model behavior. VISMOD presently supports production of contour and vector plots with geographical overlay and labeling, vertical crosssections, and animated display. VISMOD also supports user-selectable color options and contour intervals. Geographic background selection is dependent upon the model type specified to NEONS. Figure 5 is typical of VISMOD graphical output, which may be obtained for any grid-based data structure within NEONS, including VERMOD output. Other examples are presented by Leese et al. (1992, this issue).

During 1992, PROTEMS will be given a thorough exposure and evaluation when it is used to support the Data Assimilation and Model Evaluation Experiments (DAMEÉ). Several different modeling groups will conduct identical case studies and compare the results using PROTEMS.

\section{Future Plans of the Experimental Center for Mesoscale Ocean Prediction}

In keeping with the long-term goal of ECMOP, some portion of the ECMOP staff will be involved in researching, developing, and implementing capabilities into the ECMOP Modular System (EMS), to maintain a facility at the leading edge of science and technology. Direct interaction with ocean models will be the primary objective of upgrades to PROTEMS needed to achieve the operational version, EMS 1.0. PROTEMS provides only two-dimensional representations; EMS 1.0 will provide three-dimensional representations, as 
well as direct interaction with two-dimensional data displays. In addition, EMS 1.0 will give the scientist easy access to a full range of modeling support applications directly from a graphics terminal or workstation. The user will be able to devote maximum time to model evaluation and fine tuning because EMS 1.0 will provide the rest.

The focus will continue to be on compiling data sets needed for Navy ocean-modeling research and operations. By 1993, the ECMOP plans to compile a verification data set for the North Atlantic Basin covering the useful data period from 1986 to 1989 of the GEOSAT satellite. The compilation of a global-ocean verification data set should begin by 1995.

The ECMOP facility will contain a library of ocean models/modules with varying types of support. Some models/modules will be used routinely by a major segment of the user group and will be fully documented and maintained by ECMOP. Others, with narrower applicability, will have shared support between ECMOP and the originator. Those models/modules used only by a specific investigator may be present in the library with support provided by the investigator.

ECMOP is intended to meet the common research-support needs of a broad segment of the modeling community. Additionally, substantial improvement can be expected in the transition to operational users if the same methodology and technology are used (as much as possible) by those that develop and those that use the models. This has been accomplished with transition of a prototype version of the system to the Naval Oceanographic Office.

\section{Acknowledgements}

We thank J. Hovermale and T. Tsui from the Atmospheric Directorate of NRL for providing their NEONS software to enable INO to get an accelerated start on the ECMOP modular system; V. Anantharaj, H. Anand, and R. Krishnamagaru for their extra efforts in getting the early versions of the ECMOP Modular System completed; and all the participants at the 1988 ECMOP Workshop.

\section{References}

Anand, H., M.S. Foster, R. Krishnamagaru and R.M. Passi, 1991: VERMOD Capabilities: VERMOD 1.0 and Future. INO Technical Memo TM-4.

Blumberg, A.F. and G.L. Mellor, 1987: A description of a threedimensional coastal ocean circulation model. In: ThreeDimensional Coastal Ocean Models, vol. 4, N. Heape, ed., American Geophysical Union, Washington, DC, $208 \mathrm{pp}$.

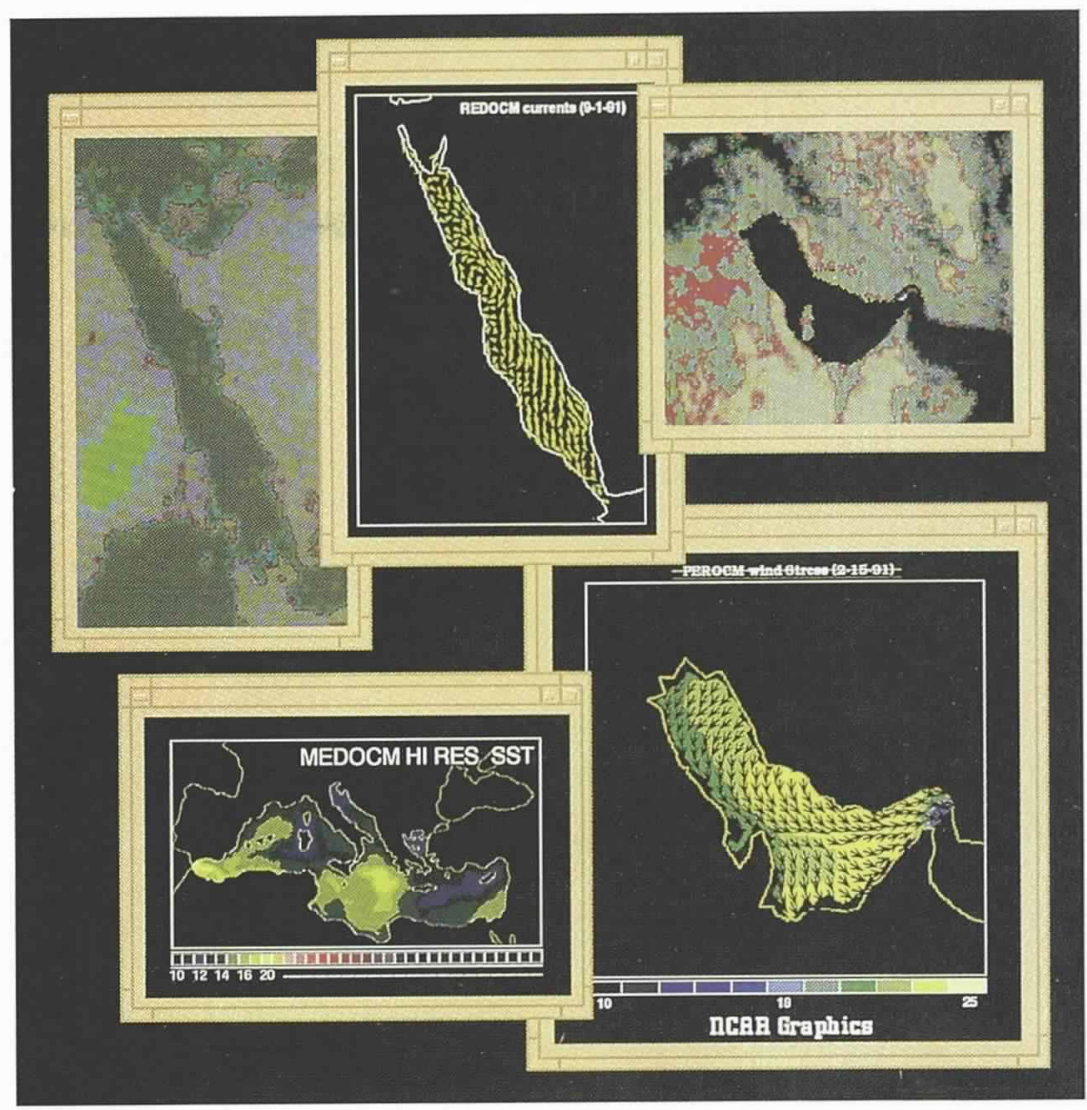

Fig. 5: Several examples of graphical output from the ECMOP visualization module demonstrating the flexibility for data-base control of model output geometries and satellite image enhancement possibilities.

Derber, J. and A. Rosati, 1989: A global oceanic data assimilation system. J. Phys. Oceanogr., 19, 1333-1347.

INO, 1988: Experimental Center for Mesoscale Ocean Prediction (ECMOP), Report on a Workshop. INO Report 89-2, University of Southern Mississippi, Long Beach, MS.

Leese, J.A., R.C. Willems and L.A. Yeske, 1992: Evaluation capability for the Navy Ocean Modeling and Prediction Program. Oceanography, 5, 55-59.

Mooers, C.N.K., A.R. Robinson and J.D. Thompson, eds., 1986: Proceedings of the Ocean Prediction Workshop. Phase I, Cambridge, MA, Phase II, Long Beach, MS.

Waters, M.P., R.G. Kelley, B.S. Carlson and M.J. Roth, 1990: Trial Ocean Prediction Experiment of the ECMOP PEDAM (GOM). Unpublished Final Report of the U.S. Naval Academy/INO Summer Research Program.

Willems, R.C., 1989: Summer colloquium on Mesoscale Ocean Science and Prediction: Model Evaluation Criteria. INO Technical Note (PROC-I), National Center for Atmospheric Research, Boulder, CO.

Wilmot, C.J., S.G. Ackleson, R.E. Davis, J.J. Feddema, K.M. Klink, D.R. Legates, J. O'Donnell and C.M. Rowe, 1985: Statistics for the Evaluation and Comparison of Models. J. Geophys. Res., 90, 8995-9005. 